\title{
Trefftz analysis for temperature rise on human skin exposed to electromagnetic waves
}

\author{
Y. Hirayama \& E. Kita \\ Graduate School of Information Sciences, Nagoya University, \\ Nagoya 464-8301, Japan
}

\begin{abstract}
This paper describes the application of Trefftz method to the temperature rise in a human skin exposed to a radiation from a cellular phone. A governing equation is given as Poisson equation. An inhomogeneous term of the equation is approximated with a polynomial function in Cartesian coordinates. The use of the approximated term transforms the original boundary-value problem to that governed with a homogeneous differential equation. The transformed problem can be solved by the traditional Trefftz formulation. Firstly, the present method is applied to a simple numerical example in order to confirm the formulation. The temperature rise in a skin exposed to a radiation is considered as a second example.

Keywords: Trefftz method, poisson equation, polynomial function.
\end{abstract}

\section{Introduction}

There has been an increasing public concern regarding the possible health effects of human exposure to an electromagnetic radiation. In Japan, there are four cellular phone carrier companies; NTT Docomo, au, Softbank (vodafone) and Willcome. They provide different types of cellular phone services. NTT Docomo, au and Softbank (vodafone) provides Code Division Multiple Access (CDMA)-type phones and Willcome Personal Handy-phone System (PHS)-type ones. The CDMA-type phones are so-called 3G-cellular phones, which are used widely around the world. The PHS-type phones operate in the $1880-1930 \mathrm{MHz}$ frequency band, which are used mainly in Japan, China, Taiwan and some other Asian countries. Since carrier companies operate different services, their health effects are also dissimilar. In this paper, we will focus on the temperature rise in a human skin exposed to the radiation. The hazardous electromagnetic field levels can be quantified analyzing the thermal response of the human body exposed to the radiation $[1,2]$. 
The temperature rise in a human skin exposed to the radiation can be defined as a boundary-value problem of a Poisson equation. Original boundary element methods are not adequate for solving an inhomogeneous differential equation such as Poisson equation. Since there exists a domain integral term derived from an inhomogeneous term of the equation in the integral equation, domain discretization is necessary. For transforming the domain integral due to a boundary one, several formulations have been presented; dual reciprocity method [3, 4], multiple reciprocity method [5, 6], radial bases function approximation [7-11], polynomial function approximation method [12], boundary point interpolation method [13], and so on.

In this paper, Trefftz method based on the collocation formulation is applied to the problem [14-16]. Since an integral equation is not necessary, the abovementioned formulations can not be applied directly for the formulation. So, we will use the polynomial function approximation method of the particular solution of the problem. The problem to be solved is governed with a Poisson equation. An inhomogeneous term of the equation is approximated with a polynomial function in Cartesian coordinates. The approximated expression of the particular solution can be derived easily. The use of the approximated particular solution transforms the boundary value problem of Poisson equation to that of Laplace equation. The boundary value problem governed with Poisson equation can be solved easily according to the traditional Trefftz formulation.

The remaining of the paper is organized as follows. In section 2 , the formulation of the problem to be solved is described. In section 3, the present method is applied to some numerical examples. The section 4 summarizes the conclusions.

\section{Formulation}

\subsection{Governing Equation and Boundary Condition [1, 2]}

For harmonically varying electromagnetic field, the temperature rise on the human skin can be calculated from the bio-heat equation

$$
C \rho \frac{\partial u}{\partial t}=K \nabla^{2} u+Q_{e m}-B\left(u-u_{b}\right),
$$

where $u$ is the temperature of the tissue, $u_{b}$ is the temperature of the blood, $K$ is the thermal conductivity of the tissue, $C$ is the heat capacity of the tissue, $B$ is the term associated with blood flow, and $Q_{e m}$ is the electromagnetic power deposition.

The electromagnetic power deposition $Q_{e m}$ is given as

$$
Q_{e m}=\rho \cdot S A R
$$

where $\rho$ and $S A R$ denote a tissue density and specific absorption rate (SAR), respectively. For harmonically varying electromagnetic field, the $S A R$ is defined as

$$
S A R=\frac{\sigma}{2 \rho}|E|^{2}
$$


where $|E|$ is the peak value of electromagnetic-field, and $\sigma$ is the conductivity of the tissue.

In addition, the boundary condition for equation (1) is given by

$$
\begin{array}{r}
u=\bar{u}, \\
q \equiv \frac{\partial u}{\partial n}=\bar{q},
\end{array}
$$

and, on the skin surface,

$$
h \cdot\left(u-u_{a}\right)=-K q
$$

where $H$ and $u_{a}$ are the convection coefficient and temperature of the air, respectively.

At the thermal steady state, $\partial u / \partial t=0$ and therefore, equation (1) is reduced to the

$$
\nabla^{2} u+b=0
$$

where

$$
b=\frac{1}{K}\left[\rho \cdot S A R-B\left(u-u_{a}\right)\right] .
$$

The boundary conditions (4) to (6) are rewritten as

$$
\alpha u+\beta q=\gamma
$$

where $\alpha, \beta$ and $\gamma$ denote the parameters derived from the boundary conditions.

\subsection{Trefftz Formulation [17]}

Now, we consider the boundary value problem defined by equations (7) and (9). An inhomogeneous term (8) is approximated with a polynomial function in Cartesian coordinates;

$$
b=c^{T} \boldsymbol{r} .
$$

In this study, the fifth-order polynomial is adopted for the function $\boldsymbol{r}$. Therefore, $\boldsymbol{c}$ and $\boldsymbol{r}$ are defined respectively as follows

$$
\begin{aligned}
\boldsymbol{c}^{T}= & \left\{c_{1}, c_{2}, \cdots, c_{21}\right\}, \\
\boldsymbol{r}^{T}= & \left\{r_{1}, r_{2}, r_{3}, \cdots, r_{21}\right\} \\
= & \left\{1, x, y, x^{2}, x y, y^{2}, x^{3}, x^{2} y, x y^{2}, y^{3}, y^{3}, x^{4}, x^{3} y,\right. \\
& \left.x^{2} y^{2}, x y^{3}, y^{4}, x^{5}, x^{4} y, x^{3} y^{2}, x^{2} y^{3}, x y^{4}, y^{5}\right\} .
\end{aligned}
$$


The use of the equation (10) transforms the original governing equation into

$$
\nabla^{2} u+c^{T} \boldsymbol{r}=0
$$

Since the term $r_{i}$ is a polynomial function, the related particular solution $u_{i}^{p}$ can be determined easily. $u_{i}^{p}$ satisfies the equation

$$
\nabla^{2} u_{i}^{p}+r_{i}=0
$$

In the Trefftz method, the homogeneous solution of the governing equation $u^{h}$ is approximated with the superposition of the related T-complete function $u_{i}^{*}$ [18]. The unknown function $u$ is approximated with the T-complete function $u_{i}^{*}$ and the particular solution $u_{i}^{p}$ as follows.

$$
u=u^{h}+c^{T} \boldsymbol{u}^{p}=\boldsymbol{a}^{T} \boldsymbol{u}^{*}+\boldsymbol{c}^{T} \boldsymbol{u}^{p}
$$

where $\boldsymbol{a}$ denotes the unknown parameter vector for approximating the homogeneous solution. Besides, $u_{i}^{*}$ and $u_{i}^{p}$ satisfy the equations

$$
\begin{aligned}
\nabla^{2} u_{i}^{*} & =0, \\
\nabla^{2} u_{i}^{p}+r_{i} & =0 .
\end{aligned}
$$

Equation (15) satisfies Eq. (7) but dose not satisfy Eq. (9). Substituting Eq. (15) into (9) leads to residual expressions:

$$
\begin{aligned}
R & =\alpha u+\beta q-\gamma \\
& =\alpha\left(\boldsymbol{a}^{T} \boldsymbol{u}^{*}+\boldsymbol{c}^{T} \boldsymbol{u}^{p}\right)+\beta\left(\boldsymbol{a}^{T} \boldsymbol{q}^{*}+\boldsymbol{c}^{T} \boldsymbol{q}^{p}\right)-\gamma \\
& =\left(\alpha \boldsymbol{u}^{*}+\beta \boldsymbol{q}^{*}\right)^{T} \boldsymbol{a}+\left(\alpha \boldsymbol{u}^{p}+\beta \boldsymbol{q}^{p}\right)^{T} \boldsymbol{c}-\gamma
\end{aligned}
$$

where

$$
\left.\begin{array}{c}
q_{i}^{*}=\frac{\partial u_{i}^{*}}{\partial \boldsymbol{n}} \\
q_{i}^{p}=\frac{\partial u_{i}^{p}}{\partial \boldsymbol{n}}
\end{array}\right\} .
$$

Satisfying the residual equations at the boundary point $P_{m}$ by means of the collocation formulation, we have

$$
\boldsymbol{K a}=\boldsymbol{f}-\boldsymbol{B} \boldsymbol{c},
$$

In the matrix $\boldsymbol{K}$, the total numbers of the rows and the columns are equal to the total number of the boundary collocation points and the T-complete functions, respectively. Therefore, we shall take more collocation points than the T-complete functions; i.e., $M>N$ and Equation (18) is solved by using the singular value decomposition method of the LAPACK software [19]. 


\subsection{Determination of parameter $c$}

The unknown parameter vector $\boldsymbol{c}$ in Eq. (18) is determined by using the iterative process. Equation (10) held at the iteration steps $(k)$ and $(k+1)$ are

$$
\begin{gathered}
b^{(k+1)}=\boldsymbol{r}^{T} \boldsymbol{c}^{(k+1)}, \\
b^{(k)}=\boldsymbol{r}^{T} \boldsymbol{c}^{(k)} .
\end{gathered}
$$

Subtracting both sides of the above equations leads to

$$
\begin{aligned}
b^{(k+1)}-b^{(k)} & =\boldsymbol{r}^{T}\left(c^{(k+1)}-c^{(k)}\right), \\
\Delta b & =\boldsymbol{r}^{T} \Delta \boldsymbol{c},
\end{aligned}
$$

where the superscript $(k)$ denotes the number of iteration.

The collocation points, which are referred as "the computing point", are placed on the boundary and within the domain. Holding Equation (19) on the computing points and arranging them in the matrix form, we have

$$
D \Delta c=f
$$

where $\boldsymbol{D}$ and $\boldsymbol{f}$ denote the coefficient matrix and vector, respectively. Equation (20) is solved for $\Delta \boldsymbol{c}$ with the singular value decomposition of LAPACK software [19]. The parameter $c$ is updated with

$$
c^{(k+1)}=\boldsymbol{c}^{(k)}+\Delta \boldsymbol{c} .
$$

The convergence criterion is defined as

$$
\eta \equiv \frac{1}{M_{c}} \sum_{i=1}^{M_{c}}\left|\Delta b\left(Q_{i}\right)\right|<\eta_{c}
$$

where $M_{c}$ and $\eta_{c}$ denote the total number of the computing points and the positive constant specified by a user, respectively. $Q_{i}$ denotes the computing points placed on the boundary and within the domain.

\section{Numerical examples}

The object under consideration is a square region of $-1 \leq x \leq 1,-1 \leq y \leq 1$ (Fig. 1). Figure 1 shows the part of a human skin. The right segment is exposed to the air and the other segments are in the body. The physical parameters are given as follows; $\rho=1010\left[\mathrm{~kg} / \mathrm{m}^{3}\right], K=0.50\left[\mathrm{~W} / \mathrm{m} \cdot{ }^{\circ}\right], B=8650\left[\mathrm{~W} / \mathrm{m}^{3 \circ}\right]$, $u_{b}=37.0\left[^{\circ}\right], h=10.5\left[\mathrm{~W} / \mathrm{m}^{3 \circ}\right]$, and $u_{a}=27.0\left[^{\circ}\right]$. 


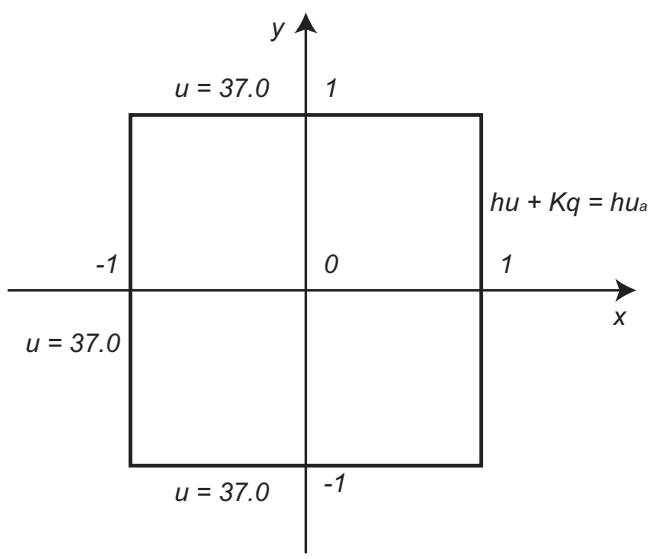

Figure 1: Numerical example.

Table 1: Numerical results.

\begin{tabular}{llc}
\hline Model & SAR & Max. Temp. Rise $\left[^{\circ}\right]$ \\
\hline Docomo SO702i & 1.35 & 0.000409 \\
Nokia 702NK2 & 0.83 & 0.000251 \\
au Talby & 0.499 & 0.000151 \\
Willcom WA007SH & 0.021 & 0.000006 \\
\hline
\end{tabular}

The parameter $S A R[\mathrm{~W} / \mathrm{kg}]$ depends on cellular phones. We will consider four cellular phones; Docomo (SO702i), au(Talby), Softbank(Nokia 702NK2), and Willcom(WA007SH), which are popular in Japan. The boundary conditions are given as

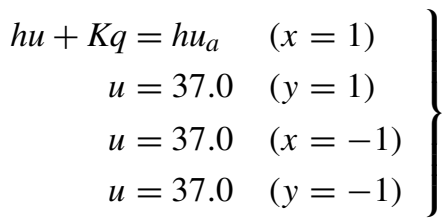

Numerical results are shown in Table 1. Distributions of the temperature rise are shown in Figs. 2 to 5. The temperature rise increases according to the increase of the SAR value. They are 3G-type cellular phones, except for Willcom(WA007SH). However, from the view-point of the rise in the human skin, we notice that Willcom(WA007SH) is the most gentle and that the au(Talby) is gentler than the other 3G-type cellular phones. 
Boundary Elements and Other Mesh Reduction Methods XXX 101

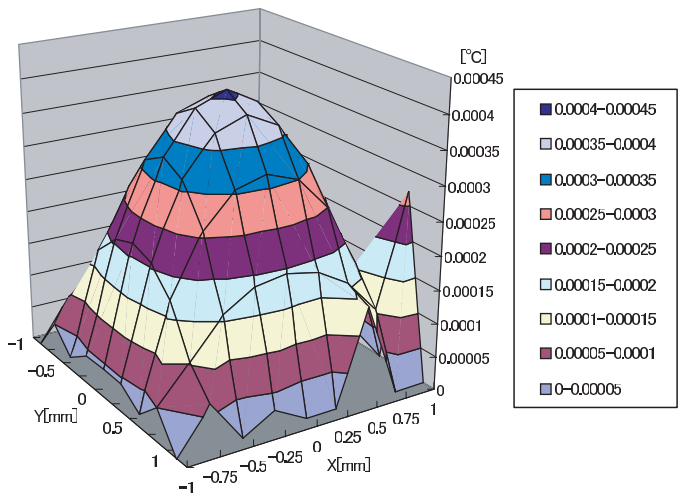

Figure 2: Docomo (SO702i).

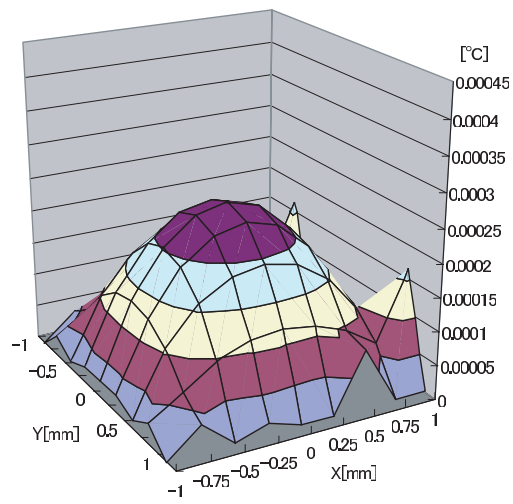

口0.0004-0.00045

口0.00035-0.0004

口0.0003-0.00035

$\square 0,00025-0.0003$

$\square 0.0002-0.00025$

$\square 0.00015-0.0002$

$\square 0.0001-0.00015$

$\square 0,00005-0.0001$

$\square 0-0,00005$

Figure 3: Softbank (Nokia 702NK2).

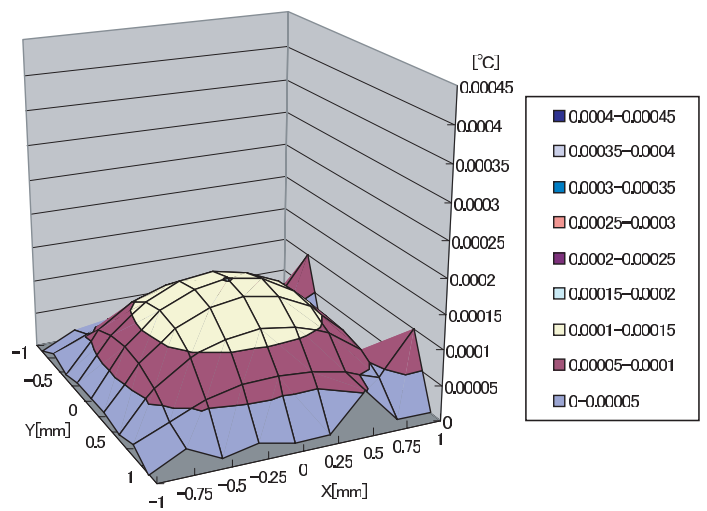

Figure 4: au (Talby). 


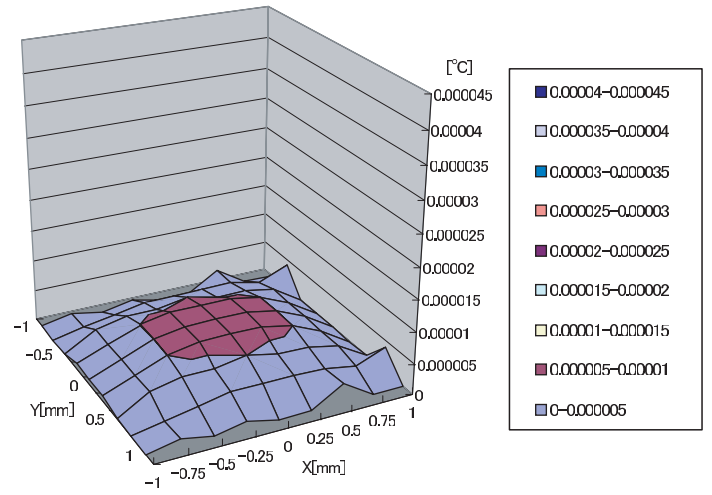

Figure 5: Willcom (WA007SH).

\section{Conclusions}

This paper describes the application of the Trefftz method to the temperature rise analysis in a human skip exposed to a radiation from a cellular phone. The governing equation is given as Poisson equation. An inhomogeneous term of the equation is approximated with a polynomial function in Cartesian coordinates. The use of the approximated term transforms the original boundary-value problem to that governed with a homogeneous differential equation. The transformed problem can be solved by the traditional Trefftz formulation. We compare four cellular phones from the view-point of the rise in the human skin; NTT Docomo (SO702i), au(Talby), Softbank(Nokia 702NK2), and Willcom(WA007SH), which are popular in Japan. They are 3G-type cellular phones, except for Willcom(WA007SH). We notice that Willcom(WA007SH) is the most gentle and that the au(Talby) is gentler than the other 3G-type cellular phones.

\section{References}

[1] A. Hirata and T. Shiozawa. Correlation of maximum temperature increase and peak SAR in the human head due to handset antennas. IEEE, Transactions on Microwave Theory and Techniques, Vol. 51, No. 7, pp. 1834-1841, 1999.

[2] D. Poljak, N. Kovac, T. Samardzioska, A. Peratta, and C. A. Brebbia. Temperature rise in the human body exposed to radiation from base station antennas. In C. A. Brebbia, editor, Boundary Elements XXVI (Proc. 26th World Conf. on BEM, Bologna, Italy, 2004), pp. 381-390. WIT Press, 2004.

[3] T. W. Partridge, C. A. Brebbia, and L. C. Wrobel. The Dual Reciprocity Boundary Element Method. Comp. Mech. Pub. / Springer Verlag, 1992.

[4] T. W. Partridge. Towards criteria for selecting approximation functions in the dual reciprocity method. Engineering Analysis with Boundary Elements, Vol. 24, No. 7, pp. 519-529, 2000. 
[5] A. J. Nowak and A. C. Neves. The Multiple Reciprocity Boundary Element Method. Comp. Mech. Pub. / Springer Verlag, 1994.

[6] A. J. Nowak. Application of the multiple reciprocity BEM to nonlinear potential problems. Engineering Analysis with Boundary Elements, Vol. 18, pp. 323-332, 1995.

[7] Z. Liu and J. G. Korvnik. Accurate solving the Poisson equation by combining multiscale radial basis functions and Gaussian quadrature. In C. A. Brebbia, A. Tadeu, and V. Popov, editors, Boundary Elements XXIV (Proc. 24th World Conf. on BEM, Sintra, Portugal, 2002), pp. 97-104. Comp. Mech. Pub., 2002.

[8] W. Chen. Some recent advances on the RBF. In C. A. Brebbia, A. Tadeu, and V. Popov, editors, Boundary Elements XXIV (Proc. 24th World Conf. on BEM, Sintra, Portugal, 2002), pp. 125-134. Comp. Mech. Pub., 2002.

[9] V. M. A. Leitao and C. M. Tiago. The use of radial basis functions for one-dimensional structural analysis problmes. In C. A. Brebbia, A. Tadeu, and V. Popov, editors, Boundary Elements XXIV (Proc. 24th World Conf. on BEM, Sintra, Portugal, 2002), pp. 165-179. Comp. Mech. Pub., 2002.

[10] B. Sarler, J. Perko, and C.S. Chen. Radial basis function collocation method solution of natural convection in porous media. International Journal of Numerical Methods for Heat and Fluid Flow, Vol. 14, No. 2, pp. 187-212, 2004.

[11] M.S. Ingber, C.S. Chen, and J.A. Tanski. A mesh free approach using radial basis functions and parallel domain decomposition for solving three dimensional diffusion equations. International Journal for Numerical Methods in Engineering, Vol. 60, pp. 2183-2201, 2004.

[12] Xu S.Q. and Kamiya N. A formulation and solution for boundary element analysis of inhomogeneous-nonlinear problem; the case involving derivatives of unknown function. Engineering Analysis with Boundary Elements, Vol. 23, No. 5/6, p. 391, 1999.

[13] G. R. Liu and Y. T. Gu. Boundary meshfree methods basd on the boundary point interpolation methods. In C. A. Brebbia, A. Tadeu, and V. Popov, editors, Boundary Elements XXIV (Proc. 24th World Conf. on BEM, Sintra, Portugal, 2002), pp. 57-66. Comp. Mech. Pub., 2002.

[14] E. Trefftz. Ein Gegenstück zum Ritzschen Verfahren. Proc. 2nd Int. Cong. Appl. Mech., Zurich, pp. 131-137, 1926.

[15] Y. K. Cheung, W. G. Jin, and O. C. Zienkiewicz. Direct solution procedure for solution of harmonic problems using complete, non-singular, Trefftz functions. Communications in Applied Numerical Methods, Vol. 5, pp. 159-169, 1989.

[16] M. Karas and A. P. Zielinski. Application of Trefftz complete functional system to stress analysis in helical spring with an arbitrary wire cross-section. Strojnicky Casopis, Vol. 49, pp. 426-437, 1998.

[17] E. Kita, Y. Ikeda, and N. Kamiya. Trefftz solution for boundary value problem of three-dimensional poisson equation. Engineering Analysis with Boundary Elements, Vol. 29, pp. 383-390, 2005. 
[18] I. Herrera. Theory of connectivity: A systematic formulation of boundary element methods. In C. A. Brebbia, editor, New Developments in Boundary Element Methods (Proc. 2nd Int. Seminar on Recent Advances in BEM, Southampton, England, 1980), pp. 45-58. Pentech Press, 1980.

[19] E. Anderson, Z. Bai, C. Bischof, J. Demmel, J. Dongarra, J. Du Croz, A. Greenbaum, S. Hammarling, A. McKenney, S. Ostrouchov, and D. Sorensen. LAPACK User's Manual. SIAM, 2.nd edition, 1995. 